\title{
Immune cells and their interactions
}

\author{
Many insights derived from the immune system apply elsewhere, as Nature's conference, "The immune system as \\ a model organ" (Boston, 7-8 November) learned. But in other respects, the immune system remains unique.
}

Boston. Half-way through a talk on lymphocyte homing and adhesion, Tim Springer (Harvard Medical School) took two dark lumps from his pocket and threw them at the projection screen. After a resounding thud, they stuck for several minutes, before sliding slowly downwards. The resemblance to the live lymphocytes he had just shown on video was startling, even if the latter normally roll before they adhere.

The relationship between lymphocytes and 'splat-balls' (as they proved subsequently to be called) may be only skin-deep. But might the parallels between the cells of the immune system and those elsewhere in the body be more than superficial? Certainly, the ease with which such cells and the cancers that affect them can be cultured have led to their intensive study.

Of course, some molecules of the immune system, like the elaborate antigenspecific receptors found on B and T cells, have no exact equivalent elsewhere. But as Dan Littman (University of California, San Francisco (UCSF)), Arthur Weiss (UCSF) and John Cambier (National Jewish Center for Immunology and Respiratory Medicine) showed, they may nevertheless induce effects very like those of other receptors when they have bound a ligand (MHC plus peptide in the case of the T-cell receptor, antigen in the case of the B-cell receptor).

In both cases, ligands induce rapid activation of an Src-family tyrosine kinase, which phosphorylates several related motifs on the receptor, forming a binding site for another tyrosine kinase called Zap-70, which in turn is activated by phosphorylation. In vivo, some of these steps may precede ligand binding, but the result is the same: a series of intracellular signals activating phospholipase $\mathrm{Cg}$, phosphatidylinositol-3-OH kinase, and the MAP kinase cascade.

More conventional receptors such as that for epidermal growth factor induce very similar events. But the T-cell receptor goes further: its effects depend on its affinity for its ligand. Too low an affinity, and the cell in question dies by apoptosis; but too high an affinity also leads to cell death or anergy. Only receptors of intermediate affinity induce positive selection. The basis for this distinction is not fully understood, but selected T-cells appear to recognize peptides that are receptor antagonists, and the thymic ligand density is also important (Michael Bevan, University of Washington).

Other systems mediate a wide range of responses in a different way. The ErbB group of receptors, for instance, are all closely related to the epidermal growth factor receptor (Yosef Yarden, Weizmann Institute, Israel). But each member of the family can form a heterodimer with any of the others, and each heterodimer has a distinct affinity for several different ligands, allowing very complex behaviour.

In other cases, closely related receptor subunits bearing identical Jak-type kinases can nevertheless phosphorylate distinct transcription factors of the Stat family (George Yancopoulos, Regeneron Pharmaceuticals). The basis for this selectivity appears to reside in the intracellular domains of the receptor subunits, which bring only the correct Stat(s) to the Jaks' attention.

Inside the cell, too, the parallels between lymphocytes and other cells are close. Numerous stimuli activate the immune transcription factor NF- $\mathrm{kB}$ by inducing the degradation of its inhibitor I-kB (David Baltimore, Massachusetts Institute of Technology (MIT)). But who would have thought that a very similar system would control dorso-ventral axis formation in Drosophila? For all its generality, however, the system is far from fully understood. As yet we can only guess why mice lacking the $\mathrm{p} 50$ subunit of NF-kB are in most respects perfectly healthy, while those lacking other subunits perish at an early age.

Sometimes, generality can be exploited. The immunosuppressants cyclosporin $A$ and FK506 interfere with T-cell activation by blocking the action of a calcium-dependent phosphatase called calcineurin. But both drugs must bind to small proteins called cyclophylins to act. Can a dimer of immunosuppressants bring together two proteins that do not normally interact, if the proteins are tagged with cyclophylins? It can, and the resulting research tool promises to be very useful (Gerald Crabtree, Stanford University Medical Institute).

Although the immune system was once thought to be unusual, in that its cells interacted in solution, it is increasingly clear that this is only partly true. Even formation of a blood clot begins with a site-specific interaction between fibronectin and the integrin receptors on platelets, triggering a network of intracellular signals that culminates in the activation of focal adhesion kinase (Joan Brugge, ARIAD Pharmaceuticals). And the rolling process studied by Springer is important precisely because it allows lymphocytes to form the interactions responsible for adhesion.

Such contacts are even more important in the bone marrow, where they direct the earliest steps of immune cell differentiation. Even there, though, it is unlikely that they involve the exact three-dimensional specificity of the interactions specifying the initial formation of mesoderm in the vertebrate embryo (Douglas Melton, Harvard) or the patterning of different cell types in the nascent spinal chord (Thomas Jessell, Columbia). As in the immune system, both secreted and cell surface cytokines are important; the finding that brain-derived neurotrophic factor directs the innervation of the vestibular apparatus, but not the inner hair cells of the ear, while the opposite is true of neurotrophin-3, underlines the specialization of such trophic interactions (RudolfJaenisch, Whitehead Institute, MIT).

Whether cells are killed by virtue of their position or the affinity of their T-cell receptor, however, the pathway by which they die is similar. But instead of being a model, the immune system in this case follows lines first uncovered in the worm Caenorhabditis elegans (Robert H. Horvitz, MIT). In this organism, 1,090 cells are formed, of which 131 die by apoptosis, a form of cellular suicide triggered by the action of a protease encoded by the ced-3 gene. In the remaining cells, the process is blocked by the ced-9 gene, whose mammalian homologue is $b c l$ 2 , and overexpression of $b c l-2$ indeed dramatically increases the number of circulating B-cells (Suzanne Cory, Walter \& Eliza Hall Institute of Medical Research).

Even here, though, the immune system holds surprises. Overexpression of $b c l-2$ does not preserve $\mathrm{T}$ cells from apoptosis (Cory). Moreover, while TNFa itself induces apoptosis in many cell types, an antibody to TNFa blocks the ability of lipopolysaccharide to rescue T-cells from the wave of death that normally limits their response to an antigen, suggesting that in this case TNFa may be anti-apoptotic (Philippa Marrack, National Jewish Center for Immunology and Respiratory Medicine).

So is the immune system a model organ? Clearly, the point can be pushed too far. It would be foolish to compare the ways in which lymphocytes and neurons store information, for example. And the parallels between AIDS (where persistent activation of non-infected cells increasingly seems to be important - Anthony Fauci, National Institutes of Health) and organotropic diseases such as hepatitis are fairly limited. But in other respects, so much of what has been learned about immune system has been or can be applied to other organs that the answer can only be yes. Nicholas Short 